\title{
In Swimming Branch, Identification of Family Burden of Families of Mentally Disabled Athletes
}

\author{
Eyyup Nacar ${ }^{1}$, M. Fatıh Karahuseyınoglu ${ }^{1}$, Baykal Karatas ${ }^{1}$, Oguzhan Altungul ${ }^{1}$ \\ ${ }^{1}$ Frrat University Sports Sciences Faculty, Elazig, Turkey \\ Correspondence: Firat University Sports Sciences Faculty, Elazıg, Turkey.
}

Received: September 19, 2017

Accepted: October 11, $2017 \quad$ Online Published: October 15, 2017

doi:10.11114/jets.v5i11.2706

URL: https://doi.org/10.11114/jets.v5i11.2706

\begin{abstract}
The families of individuals with down-syndrome, autism, and mental problem who need for special requirements experience physical problems, tiredness, and antisocial life, which bring additional cost to family budget, from time to time due to difficulties of their children The aim of this study is to identify family burdens charged by kids with special education needs, and in terms of which variables it becomes different.

While the study was designed by the part literature", obtained from scientific resources, personal information form, in which the demographic information of the families of kids with special needs take place, and the results of family burden assessment score, developed by Sarı and Basbakkal (2008) toward the aim of the study and consisted of 43 questions and 6 sub dimensions, were taken into consideration. In identifying family burdens, with moving from the assumption that mothers have dominant effect, the study was administered to the mothers

Majority of the families participating in the study are housewife. Total mean score obtained from family burden scale were 127 in the range of 45 to 215 points. In the study carried out, it was revealed that economic burden resulted from not employed housewives; and among the factors affecting perceived inabilities of mothers with disabled child, the concerns mothers experience toward the future of their children were very difficult case for the mothers.

However, families experience a concern in the form of "After my death, who will look after my child? .In the study carried out, it revealed that this case led the burdens of perceived inability to increase; and that the families tried to cope with this case, which causes concern for their kids with special needs.

In the world, the sincere of the mothers facing with this kind of difficulties in the face of these cases is a great chance for kids with special needs.
\end{abstract}

Keywords: family of disabled athlete, disabled athlete, family burden, disabled swimmer

\section{Introduction}

Burden is subjective perceptions or individual reactions a person/mother giving care to a disabled child experiences, while giving care to it. The feelings caregiver perceives are a concept encompassing her physical health, economic status, and social life (Chou, 2000).

The concept of family burden was first voiced in 1960s and defined as negative costs those having a mental disorder create for their families. In Western Australia (WA), governmental organ providing resource for disabled people is Committee of Disability Services. This committee has two separate financial flows supporting mentally disabled individuals for their living out of parental house: United Application Process and Community Life Plan (Naomi Catal. 2014) In Turkey, this service is carried out by Ministry of Family and Social Policies. Family burden can be also defined as all difficulties family experiences as a result of illness of a person (Sarı and Başbakkal, 2008).

Living with a mentally unable child in family and giving care to it form a burden sense on family members. . Fishman and Wolf (1991) identified that the parents having child with Autism and Down Syndrome experienced stress in higher rate compared to the parents of normal children and, psychological states of especially mothers were negatively affected .( Fishman S, Wolf L. (1991).

They suggested that mothers were negatively affected from the looks turning to them and that they felt anger against these people. Another factor affecting emotional burden of families having mentally unable was stated to be concern 
they experience toward the future of their children. Families experience anxiety in the form of "Who will look after my child after my death?" and this leads emotional burden of the families to increase (Sar1 et al. 2006).

The families that participated in the study by Alelson (1999) expressed that disabled children needed help in the areas of meal preparation, personal care, administering medication, bathing, protection from danger, clothing, dental care, toilet, and feeding, cloth replacing. The same families also expressed that because they viewed their families in danger, wanted to reserve time for the healthy sibling, needed holiday and shopping, they wanted aid for relieving care (Abelson. 1999).

In this study, the concept of family burden was researched and defined through the families giving care the kids with special education needs. The kids with special education needs are the individuals lifelong supported by their family from different aspects. Therefore, they have some positive and negative effects on the life of family giving care. In general, inabilities that cannot be corrected, that shows continuity, and that cannot be changed limit the functions of the family giving care, and, thus, causes uneasiness in the family. These children, due to lifelong experienced daily care needs and often experienced behavioral problems, increase difficulties of their parents (Kaner, 2004).

\section{Material and Method}

The study aiming to examine the burdens of disabled individuals to their family consists of the first part, developed by providing literature support, and the second part, where family burdens are determined. The individuals participating in the study were necessarily informed about the aim and method of the study and, with the people who consented, data collecting process were continued. While the family burdens of mentally disabled individuals were identified, the assumption that mothers are effective in identifying this burden was moved from and the study was designed on mothers.

"Family Burden Assessment Scale" toward identifying the burdens of disabled individuals developed by Sarı and Basbakkal, consists of 43 questions. The scale has 6 sub dimensions expressed by the titles such as "Economic Burden", "Perceived Inability", "Social Burden", "Physical Burden", "Emotional Burden", and "Time Requirement". Cronbach alpha internal consistency coefficient of the scale was earlier found 0.92 and the coefficient identified for this study is 0.86 .

From 5-point Likert type scale, among the scores obtained by adding points between 1 and $5(1=$ never, $2=$ rarely, $3=$ sometimes, $4=$ mostly/often, and $5=$ always ), total points of minimum 43 and maximum 215 can be taken. Intersection point of Family Burden Assessment Scale was identified by those developing the scale as 97 points. The sensitivity of scale for 97 points is $84.1 \%$ and its specificity is $90.9 \%$. According to this result, a classification can be made in the form of that the families, whose score of family burden assessment scale is 97 points and over, have burden, while the families having the score less than 97 do not have family burden Sarı and Basbakkal, 2008)

In this study percentage and frequency tables on the other hand, in the comparisons' more than two, the Anova test results have been taken consideration. An assessment was conducted about between which groups the discussion takes place, taking into consideration the results of Tukey Test. Considering the data obtained, as a results of general evaluations and deductions, with scientific based "Conclusion and Suggestions", it was aimed that the study provides information for more detailed research.

\section{Findings}

Table 1. Table of Occupational and Educational Status

\begin{tabular}{lll}
\hline Occupation & $\mathrm{n}$ & $\%$ \\
Housewife & 53 & 69.7 \\
Wage Labor & 6 & 7.9 \\
Public Servant & 13 & 17.1 \\
Other Occupation & 4 & 5.3 \\
\hline Educational Status & $\mathrm{n}$ & $\%$ \\
Primary School & 31 & 40.8 \\
Secondary School & 26 & 34.2 \\
Higher Education & 19 & 25.0 \\
\hline Total & 76 & 100.0
\end{tabular}

When the study is carried out on the mothers, all participants are women. When regarded to the occupation group of 
mother, it was seen that $69.7 \%$ were housewife, $7.9 \%$, wage labor; $17.1 \%$, public servant; and $5.3 \%$, from the other occupational groups. When educational statuses of mother are examined, $40.8 \%$ of them completed primary school; $34.2 \%$, secondary school; and $25.8 \%$, higher education.

Table 2. Descriptive Characteristics of Disabled Athletes

\begin{tabular}{lll}
\hline Gender & $\mathrm{n}$ & $\%$ \\
Female & 28 & 36,8 \\
Male & 48 & 63,2 \\
\hline Age & $\mathrm{n}$ & $\%$ \\
Ages 0-10 & 2 & 2,6 \\
Ages 11-20 & 44 & 57,9 \\
Age 21 age and over & 30 & 39,5 \\
\hline Disability State & $\mathrm{n}$ & $\%$ \\
Mentally Disables & 39 & 51,3 \\
Autistic & 17 & 22,4 \\
With Down Syndrome & 20 & 26,3 \\
\hline Sate of Formation of Disability & $\mathrm{n}$ & $\%$ \\
In mother's womb & 51 & 67,1 \\
During delivery & 10 & 13,2 \\
After Accident -Disease & 15 & 19,7 \\
\hline Years of Making Sports (Sports & $\mathrm{n}$ & $\%$ \\
Age) & 13 & 17,1 \\
1-3 years & 19 & 25,0 \\
3-5 years & 44 & 57,9 \\
5-8 year & 76 & 100 \\
\hline Total &
\end{tabular}

In Table 2, when the descriptive characteristics of mentally disabled children are examined, $42.4 \%$ of them are female and $57.6 \%$ are male. When regarded to their pasts of sports, it is seen that $17.1 \%$ of them do swimming sports for $1-3$ years; $25.1 \%$, 3-5 years; and 57.9\%, 5-8 years and over. When the disability case of special athletes are regarded, 51.3\% is mentally disabled; $22.4 \%$, autistic; and $26.3 \%$, with down syndrome. When regarded to the ages of athletes; $2.6 \%$ are in the range of ages $6-10 ; 57.9 \% \mathrm{i}$ ages $11-20 ; 39.5 \%$, ages 21 and over. To the guardians of athletes participating in the survey when disability state is asked, it is seen that $67.1 \%$ of them became disable in mother's womb; $13.2 \%$, during delivery; and $19.7 \%$ on the reason for accident-disease.

Table 3. General Mean of Family Burden

\begin{tabular}{lllll}
\hline General Mean & Scale Mean & Maximum & Minimum & Standard Deviation \\
127.36 & 2.96 & 211 & 54 & 39.44 \\
\hline
\end{tabular}

When regarded to general means of the families, the families of athletes have general mean of 127.36.

Table 4. Mean of Family Burden Sub factors

\begin{tabular}{llllll}
\hline & $\begin{array}{l}\text { General } \\
\text { Mean }\end{array}$ & $\begin{array}{l}\text { Scale } \\
\text { Mean }\end{array}$ & Maximum & Minimum & $\begin{array}{l}\text { Standard } \\
\text { Deviation }\end{array}$ \\
Economic Burden & 18.80 & 3.13 & 30 & 6 & 7.66119 \\
Perceived Inability & 31.21 & 3.90 & 40 & 9 & 8.60436 \\
Social Burden & 14.72 & 2.61 & 30 & 6 & 7.57293 \\
Physical Burden & 12.38 & 2.47 & 25 & 5 & 6.80386 \\
Emotional Barden & 26.64 & 2.47 & 55 & 11 & 11.74927 \\
Time Requirement & 23.60 & 3.37 & 35 & 7 & 6.88008 \\
\hline
\end{tabular}

When the mean of family burden sub factors are regarded to, the highest mean score is seen to be on "Perceived Inability" and the lowest mean score, on "Physical Burden".

Comparison of the descriptive characteristics of the disabled children and their families with the mean score of family burden assessment scale. 
Table 5. Comparison of occupation of families with the mean score of family burden assessment scale

\begin{tabular}{|c|c|c|c|c|c|c|}
\hline $\begin{array}{l}\text { Sub factor of } \\
\text { occupation }\end{array}$ & Occupation & $\mathrm{n}$ & Mean & SD & $\mathrm{f}$ & $\mathrm{p}$ \\
\hline \multirow{5}{*}{$\begin{array}{l}\text { Economic } \\
\text { Burden }\end{array}$} & Housewife & 53 & 3.3522 & .16976 & \multirow{5}{*}{2.710} & \multirow{5}{*}{.051} \\
\hline & Wage labor & 6 & 3.0000 & .54603 & & \\
\hline & Public Servant * & 13 & 2.2692 & .28529 & & \\
\hline & Other Occupation & 4 & 3.2500 & .85662 & & \\
\hline & Total & 76 & 3.1338 & .14647 & & \\
\hline \multirow{5}{*}{$\begin{array}{l}\text { Perceived } \\
\text { Inability }\end{array}$} & Housewife $*$ & 53 & 4.1108 & .14684 & \multirow{5}{*}{2.886} & \multirow{5}{*}{.041} \\
\hline & Wage labor & 6 & 3.7917 & .25345 & & \\
\hline & Public Servant & 13 & 3.1923 & .30277 & & \\
\hline & Other Occupation & 4 & 3.5938 & .38654 & & \\
\hline & Total & 76 & 3.9013 & .12337 & & \\
\hline \multirow{5}{*}{ Social Burden } & Housewife & 53 & 3.0692 & .25856 & \multirow{5}{*}{4.611} & \multirow{5}{*}{.005} \\
\hline & Wage labor & 6 & 1.3333 & .19720 & & \\
\hline & Public Servant & 13 & 1.7564 & .26191 & & \\
\hline & Other Occupation & 4 & 1.2500 & .25000 & & \\
\hline & Total & 76 & 2.6118 & .20291 & & \\
\hline \multirow{5}{*}{ Physical Burden } & Housewife & 53 & 2.7962 & .19113 & \multirow{5}{*}{3.934} & \multirow{5}{*}{.012} \\
\hline & Wage labor & 6 & 1.5000 & .27689 & & \\
\hline & Public Servant & 13 & 1.9692 & .32013 & & \\
\hline & Other Occupation & 4 & 1.3500 & .17078 & & \\
\hline & Total & 76 & 2.4763 & .15609 & & \\
\hline \multirow{5}{*}{$\begin{array}{l}\text { Emotional } \\
\text { Burden }\end{array}$} & Housewife & 53 & 2.6604 & .15470 & \multirow{5}{*}{3.213} & \multirow{5}{*}{.028} \\
\hline & Wage labor & 6 & 1.8485 & .34069 & & \\
\hline & Public Servant & 13 & 1.9371 & .18681 & & \\
\hline & Other Occupation & 4 & 1.7045 & .22074 & & \\
\hline & Total & 76 & 2.4222 & .12252 & & \\
\hline \multirow{5}{*}{$\begin{array}{l}\text { Time } \\
\text { Requirement }\end{array}$} & Housewife & 53 & 3.5876 & .12668 & \multirow{5}{*}{3.319} & \multirow{5}{*}{.025} \\
\hline & Wage labor & 6 & 2.8333 & .33486 & & \\
\hline & Public Servant & 13 & 3.0000 & .31821 & & \\
\hline & Other Occupation & 4 & 2.5357 & .14725 & & \\
\hline & Total & 76 & 3.3722 & .11274 & & \\
\hline
\end{tabular}

When occupation of families are compared with the physical burden, social burden, emotional burden, social burden, and time requirement, which are sub factors of family burden assessment scale, any significant difference could not be found ( $p>0.05$ ). In the comparison of the mean scores of economic burden and perceived inability, a statistically significant difference was found $(\mathrm{p}<0.05)$. Advanced analysis was made to find between which groups there is a difference.

Making inter-group advanced analysis of economic burden, it was found that there was an inter-group difference between housewives and public servants $(\mathrm{p}<0.051)$ and that there was an inter-group difference of perceived inability between public servants and housewives $(\mathrm{p}<0.041)$. 
Table 6. Comparison of educational status of families with the mean score of family burden assessment scale

\begin{tabular}{|c|c|c|c|c|c|c|}
\hline Sub Factors & Education & $\mathrm{n}$ & Mean & SD & $\mathrm{f}$ & $\mathrm{p}$ \\
\hline \multirow{4}{*}{ Economic Burden } & Primary School & 31 & 3.4731 & .19509 & 2.364 & .101 \\
\hline & Secondary School & 26 & 3.0513 & .30011 & & \\
\hline & Higher Education & 19 & 2.6930 & .24723 & & \\
\hline & Total & 76 & 3.1338 & .14647 & & \\
\hline \multirow{4}{*}{ Perceived Inability } & Primary School* & 31 & 4.4032 & .16907 & 8.098 & .001 \\
\hline & Secondary School * & 26 & 3.7596 & .19001 & & \\
\hline & Higher Education* & 19 & 3.2763 & .24754 & & \\
\hline & Total & 76 & 3.9013 & .12337 & & \\
\hline \multirow{4}{*}{ Social Burden } & Primary School* & 31 & 3.3333 & .38482 & 7.508 & .001 \\
\hline & Secondary School & 26 & 2.5705 & .26184 & & \\
\hline & Higher Education* & 19 & 1.4912 & .19010 & & \\
\hline & Total & 76 & 2.6118 & .20291 & & \\
\hline \multirow{4}{*}{ Physical Burden } & Primary School* & 31 & 3.0581 & .26088 & 5.806 & .005 \\
\hline & Secondary School* & 26 & 2.2231 & .24641 & & \\
\hline & Higher Education* & 19 & 1.8737 & .22332 & & \\
\hline & Total & 76 & 2.4763 & .15609 & & \\
\hline \multirow{4}{*}{ Emotional Burden } & Primary School* & 31 & 2.8387 & .18586 & 6.009 & .004 \\
\hline & Secondary School & 26 & 2.3566 & .22692 & & \\
\hline & Higher Education* & 19 & 1.8325 & .15172 & & \\
\hline & Total & 76 & 2.4222 & .12252 & & \\
\hline \multirow{4}{*}{ Time Requirement } & Primary School & 31 & 3.5622 & .16941 & 1.610 & .207 \\
\hline & Secondary School & 26 & 3.3791 & .20046 & & \\
\hline & Higher Education & 19 & 3.0526 & .22018 & & \\
\hline & Total & 76 & 3.3722 & .11274 & & \\
\hline
\end{tabular}

When educational status of families are compared with the mean scores of physical burden and time requirement, which are sub factors of family burden assessment scale, any significant difference could not be found ( $p>0.05$ ).

In comparison of the mean scores of perceived inability, social burden, physical burden, and emotional burden, a statistically significant difference was found $(\mathrm{p}<0.05)$. Advanced analysis was made to identify between which groups there was a difference. As a result of advanced analysis made, between educational groups of perceived inability, a difference was found between primary school-graduated family groups and secondary school and higher education-graduated ones ( $\mathrm{p}<.001)$. In inter- groups of sub group of social burden, a difference was found between primary school and higher education $(\mathrm{p}<0.001)$

Table 7. Comparison of Gender Axis Family Burden Mean Scores

\begin{tabular}{|c|c|c|c|c|c|c|}
\hline $\begin{array}{l}\text { Sub Factor of } \\
\text { the Scale }\end{array}$ & Gender & $\mathrm{n}$ & Mean & $\mathrm{SD}$ & $\mathrm{f}$ & $\mathrm{p}$ \\
\hline Economic & Female & 28 & 3.3631 & .27055 & \multirow{2}{*}{4.326} & \multirow{2}{*}{.041} \\
\hline Burden & Male & 48 & 3.0000 & .16923 & & \\
\hline \multirow{2}{*}{$\begin{array}{l}\text { Perceived } \\
\text { Inability }\end{array}$} & Female & 28 & 4.1518 & .20223 & \multirow{2}{*}{.865} & \multirow{2}{*}{.365} \\
\hline & Male & 48 & 3.7552 & .15336 & & \\
\hline \multirow{2}{*}{ Social Burden } & Female & 28 & 2.8274 & .46144 & \multirow{2}{*}{2.560} & \multirow{2}{*}{.114} \\
\hline & Male & 48 & 2.4861 & .17822 & & \\
\hline \multirow{2}{*}{ Physical Burden } & Female & 28 & 2.4786 & .24653 & \multirow{2}{*}{1.664} & \multirow{2}{*}{.201} \\
\hline & Male & 48 & 2.4750 & .20296 & & \\
\hline Emotional & Female & 28 & 2.5974 & .22402 & \multirow{2}{*}{3.850} & \multirow{2}{*}{.054} \\
\hline Burden & Male & 48 & 2.3201 & .14320 & & \\
\hline Time & Female & 28 & 3.4184 & .20215 & \multirow{2}{*}{.410} & \multirow{2}{*}{.524} \\
\hline Requirement & Male & 48 & 3.3452 & .13557 & & \\
\hline
\end{tabular}

When the gender of special athletes is compared with mean scores of sub factors of family burden assessment scale, a significant difference could not be found $(\mathrm{p}>0.05)$. 
Table 8. Comparison of family burden mean scores according to the number of sports doing years

\begin{tabular}{|c|c|c|c|c|c|c|}
\hline Sub Factors & Sports Year & $\mathrm{n}$ & Mean & SD & $\mathrm{f}$ & $\mathrm{p}$ \\
\hline \multirow{4}{*}{$\begin{array}{l}\text { Economic } \\
\text { Burden }\end{array}$} & $1-3$ & 13 & 3.1795 & .28399 & \multirow{4}{*}{2.256} & \multirow{4}{*}{.112} \\
\hline & $3-5$ & 19 & 2.6140 & .26523 & & \\
\hline & $5-8$ & 44 & 3.3447 & .20372 & & \\
\hline & Total & 76 & 3.1338 & .14647 & & \\
\hline \multirow{4}{*}{$\begin{array}{l}\text { Perceived } \\
\text { Inability }\end{array}$} & $1-3$ & 13 & 4.0192 & .24752 & \multirow{4}{*}{.586} & \multirow{4}{*}{.559} \\
\hline & $3-5$ & 19 & 3.6711 & .26130 & & \\
\hline & $5-8$ & 44 & 3.9659 & .16655 & & \\
\hline & Total & 76 & 3.9013 & .12337 & & \\
\hline \multirow{4}{*}{ Social Load } & $1-3$ & 13 & 3.1795 & .88523 & \multirow{4}{*}{.975} & \multirow{4}{*}{.382} \\
\hline & $3-5$ & 19 & 2.2982 & .26138 & & \\
\hline & $5-8$ & 44 & 2.5795 & .20941 & & \\
\hline & Total & 76 & 2.6118 & .20291 & & \\
\hline \multirow{4}{*}{$\begin{array}{l}\text { Physical } \\
\text { Load }\end{array}$} & $1-3$ & 13 & 2.2462 & .34206 & \multirow{4}{*}{.446} & \multirow{4}{*}{.642} \\
\hline & $3-5$ & 19 & 2.3474 & .27481 & & \\
\hline & $5-8$ & 44 & 2.6000 & .22185 & & \\
\hline & Total & 76 & 2.4763 & .15609 & & \\
\hline \multirow{4}{*}{$\begin{array}{l}\text { Emotional } \\
\text { Load }\end{array}$} & $1-3$ & 13 & 2.4266 & .27512 & \multirow{4}{*}{1.124} & \multirow{4}{*}{.331} \\
\hline & $3-5$ & 19 & 2.1148 & .15851 & & \\
\hline & $5-8$ & 44 & 2.5537 & .18191 & & \\
\hline & Total & 76 & 2.4222 & .12252 & & \\
\hline \multirow{4}{*}{$\begin{array}{l}\text { Time } \\
\text { Requirement }\end{array}$} & $1-3$ & 13 & 3.7473 & .21285 & \multirow{4}{*}{1.66} & \multirow{4}{*}{.194} \\
\hline & $3-5$ & 19 & 3.1053 & .20316 & & \\
\hline & $5-8$ & 44 & 3.3766 & .15942 & & \\
\hline & Total & 76 & 3.3722 & .11274 & & \\
\hline
\end{tabular}

When the time the special athletes do swimming sports is compared with the mean score of family burden assessment scale, any significant difference could not be found. $(p>0.05)$.

Table 9. According to disability states of athletes, comparison of the mean scores of family burden

\begin{tabular}{|c|c|c|c|c|c|c|}
\hline Sub Factors & Disability Group & $\mathrm{n}$ & Mean & SD & $\mathrm{f}$ & $\mathrm{p}$ \\
\hline & Mental & 39 & 3.2179 & .21078 & \multirow{4}{*}{.365} & \multirow{4}{*}{.695} \\
\hline Economic & Autistic & 17 & 2.9020 & .28621 & & \\
\hline \multirow[t]{2}{*}{ Burden } & Down Syndrome & 20 & 3.1667 & .29444 & & \\
\hline & Total & 76 & 3.1338 & .14647 & & \\
\hline & Mental & 39 & 4.0417 & .19576 & \multirow{4}{*}{1.054} & \multirow{4}{*}{.354} \\
\hline Perceived & Autistic & 17 & 3.5882 & .20943 & & \\
\hline \multirow[t]{2}{*}{ Inability } & Down Syndrome & 20 & 3.8938 & .20263 & & \\
\hline & Total & 76 & 3.9013 & .12337 & & \\
\hline \multirow{4}{*}{ Social Burden } & Mental & 39 & 2.8846 & .34598 & \multirow{4}{*}{1.287} & \multirow{4}{*}{.282} \\
\hline & Autistic & 17 & 2.5784 & .28300 & & \\
\hline & Down Syndrome & 20 & 2.1083 & .26909 & & \\
\hline & Total & 76 & 2.6118 & .20291 & & \\
\hline \multirow{4}{*}{ Physical Burden } & Mental & 39 & 2.4667 & .21159 & \multirow{4}{*}{.109} & \multirow{4}{*}{.897} \\
\hline & Autistic & 17 & 2.6000 & .37652 & & \\
\hline & Down Syndrome & 20 & 2.3900 & .29717 & & \\
\hline & Total & 76 & 2.4763 & .15609 & & \\
\hline \multirow{4}{*}{$\begin{array}{l}\text { Emotional } \\
\text { Burden }\end{array}$} & Mental & 39 & 2.5641 & .18365 & \multirow{4}{*}{.779} & \multirow{4}{*}{.463} \\
\hline & Autistic & 17 & 2.1979 & .20584 & & \\
\hline & Down Syndrome & 20 & 2.3364 & .24206 & & \\
\hline & Total & 76 & 2.4222 & .12252 & & \\
\hline & Mental & 39 & 3.4139 & .15795 & \multirow{4}{*}{.783} & \multirow{4}{*}{.461} \\
\hline Tine & Autistic & 17 & 3.5378 & .17900 & & \\
\hline \multirow[t]{2}{*}{ Requirement } & Down Syndrome & 20 & 3.1500 & .25774 & & \\
\hline & Total & 76 & 3.3722 & .11274 & & \\
\hline
\end{tabular}

When special cases of athletes are compared with mean scores of sub factors of family burden assessment scale, any significant difference could not be found ( $p>0.05)$.

Table 10. Comparison of family burden mean scores according to the ages of athletes

\begin{tabular}{|c|c|c|c|c|c|c|}
\hline Sub Factors & Age & $\mathrm{n}$ & Mean & SD & $\mathrm{f}$ & $\mathrm{p}$ \\
\hline
\end{tabular}




\begin{tabular}{|c|c|c|c|c|c|c|}
\hline \multirow{4}{*}{ Economic Burden } & Ages $0-10$ & 2 & 3.7500 & .41667 & \multirow{4}{*}{1.397} & \multirow{4}{*}{.254} \\
\hline & Ages 11-20 & 44 & 2.9318 & .18688 & & \\
\hline & Age 21 and over & 30 & 3.3889 & .24295 & & \\
\hline & Total & 76 & 3.1338 & .14647 & & \\
\hline \multirow{4}{*}{ Perceived Inability } & Ages $0-10$ & 2 & 4.1875 & .06250 & \multirow{4}{*}{.689} & \multirow{4}{*}{.505} \\
\hline & Ages 11-20 & 44 & 3.7784 & .16200 & & \\
\hline & Age 21 and over & 30 & 4.0625 & .20198 & & \\
\hline & Total & 76 & 3.9013 & .12337 & & \\
\hline \multirow{4}{*}{ Social Burden } & Ages $0-10$ & 2 & 3.0000 & .33333 & \multirow{4}{*}{.848} & \multirow{4}{*}{.433} \\
\hline & Ages 11-20 & 44 & 2.3864 & .18092 & & \\
\hline & Age 21 and over & 30 & 2.9167 & .43840 & & \\
\hline & Total & 76 & 2.6118 & .20291 & & \\
\hline \multirow{4}{*}{ Physical Burden } & Ages 0-10 & 2 & 2.7000 & .30000 & \multirow{4}{*}{.276} & \multirow{4}{*}{.760} \\
\hline & Ages 11-20 & 44 & 2.3773 & .20462 & & \\
\hline & Age 21 and over & 30 & 2.6067 & .25917 & & \\
\hline & Total & 76 & 2.4763 & .15609 & & \\
\hline \multirow{4}{*}{ Emotional Burden } & Ages $0-10$ & 2 & 2.7727 & .22727 & \multirow{4}{*}{1.192} & \multirow{4}{*}{.309} \\
\hline & Ages 11-20 & 44 & 2.2624 & .14672 & & \\
\hline & Age 21 and over & 30 & 2.6333 & .21978 & & \\
\hline & Total & 76 & 2.4222 & .12252 & & \\
\hline \multirow{4}{*}{ Time Requirement } & Ages $0-10$ & 2 & 3.5714 & .28571 & \multirow{4}{*}{.322} & \multirow{4}{*}{.726} \\
\hline & Ages 11-20 & 44 & 3.2955 & .13140 & & \\
\hline & Age 21 and over & 30 & 3.4714 & .21169 & & \\
\hline & Total & 76 & 3.3722 & .11274 & & \\
\hline
\end{tabular}

When ages of special athletes are compared with mean scores of sub factors of family burden assessment scale, any significant difference could not be found ( $p>0.05)$.

Table 11. Comparison of family burden mean scores according to the disability times of athletes

\begin{tabular}{|c|c|c|c|c|c|c|}
\hline Sub Factors & Disability Times & $\mathrm{n}$ & Mean & SD & $\mathrm{f}$ & $\mathrm{p}$ \\
\hline \multirow{4}{*}{ Economic Burden } & In mother's womb & 51 & 3.0033 & .16729 & \multirow{4}{*}{1.179} & \multirow{4}{*}{.313} \\
\hline & During delivery & 10 & 3.1333 & .40885 & & \\
\hline & Result of Accident -Disease & 15 & 3.5778 & .39065 & & \\
\hline & Total & 76 & 3.1338 & .14647 & & \\
\hline \multirow{4}{*}{ Perceived Inability } & In mother's womb & 51 & 3.8039 & .14890 & \multirow{4}{*}{.658} & \multirow{4}{*}{.521} \\
\hline & During delivery & 10 & 4.0375 & 31072 & & \\
\hline & Result of Accident -Disease & 15 & 4.1417 & .30857 & & \\
\hline & Total & 76 & 3.9013 & .12337 & & \\
\hline \multirow{4}{*}{ Social Burden } & In mother's womb & 51 & 2.6144 & .27330 & \multirow{4}{*}{.172} & \multirow{4}{*}{.843} \\
\hline & During delivery & 10 & 2.3500 & .32150 & & \\
\hline & Result of Accident -Disease & 15 & 2.7778 & .40128 & & \\
\hline & Total & 76 & 2.6118 & .20291 & & \\
\hline \multirow{4}{*}{ Physical Burden } & In mother's womb & 51 & 2.4196 & .18963 & \multirow{4}{*}{.135} & \multirow{4}{*}{.874} \\
\hline & During delivery & 10 & 2.6200 & .39379 & & \\
\hline & Result of Accident -Disease & 15 & 2.5733 & .39466 & & \\
\hline & Total & 76 & 2.4763 & .15609 & & \\
\hline \multirow{4}{*}{ Emotional Burden } & In mother's womb & 51 & 2.3547 & 2.3547 & \multirow{4}{*}{1.368} & \multirow{4}{*}{.261} \\
\hline & During delivery & 10 & 2.1818 & 2.1818 & & \\
\hline & Result of Accident -Disease & 15 & 2.8121 & 2.8121 & & \\
\hline & Total & 76 & 2.4222 & 2.4222 & & \\
\hline \multirow{4}{*}{ Time Requirement } & In mother's womb & 51 & 3.2437 & .13976 & \multirow{4}{*}{1.372} & \multirow{4}{*}{.260} \\
\hline & During delivery & 10 & 3.5714 & .18443 & & \\
\hline & Result of Accident -Disease & 15 & 3.6762 & .28417 & & \\
\hline & Total & 76 & 3.3722 & .11274 & & \\
\hline
\end{tabular}

How and when the disability states of athletes occurs are compared with mean scores of sub factors of family burden assessment scale, any significant difference could not be found ( $p>0.05)$.

\section{Discussion and Conclusion}

In the study, it was studied whether or not there was burden on the families of special children (mental autism, Down syndrome) doing swimming sports. With the results emerging in the light of data, being able to meet family burden of families having special children doing sports and guiding specialists about approaching these families were aimed. 
In comparison of the mean FBAS (Family Burden Assessment Scale) with occupation of families, when the physical burden, social burden, emotional burden, and time requirement, among sub factors of family burden assessment scale, are compared, any significant difference could not be found. Making inter-group advanced analysis of economic burden and perceived inability, it can be thought that inter-group difference is present in housewives and public servants and that although there are less number of public servants, public servants can easily overcome the cases depending on economic burden, due to the fact they receive a salary from the government every month and in terms of their being sufficient to their children. Economic burden of housewives may result from not being employed of them and, among the factors affecting perceived inability of mothers with disabled child, there is a concern that mothers experience about the future of their children. Mothers experience a concern that "Who will look after my children after my death?" and it can be said that this case lead the burdens of perceived inability to rise. In the study of Sivri (2012), [it is seen] that the mothers having mentally unable children perceive family stress. That family In the study of identifying social aid and family burden, it is seen that burden turns out considerably high and has the highest mean emotional burden. Öztürk (2011), in the study he carried out, could not find any significant difference in comparison of occupation of families with the mean scores of family burden assessment score. There is a parallelism between our study and that of Öztürk (2011) in terms of physical burden, social burden, emotional burden, and time requirement.

In comparison of educational status of families with the mean scores of perceived inability, social burden, physical burden, and emotional burden, among sub factors of family burden assessment scale, it was found that there was a statistically significant difference. In the sub groups of perceived inability, physical burden, and emotional burden, it was found that there was a difference between the families graduated from primary school and the families graduated from the secondary school and higher education, while among the subgroups of social burden, there was difference between hose graduated from primary school and higher education. In this case, it can be said that the perceived inability of the families graduated from primary is higher compared to the families graduated from the secondary school and higher education in terms of physical burden and emotional burden. When we deal with in terms of social burden, it can be said that social burden of the families graduated from higher education are less compared to primary school-graduated families. In the study, carried out by Öztürk (2002), in comparison of educational status of the families and the mean scores of family burden assessment scale, it revealed that there was not any statistically significant difference. Although it was not in the same direction with our study, in the study carried out by Bildirici (2014), a statistically significant difference between the total score of family burden assessment and mean scores of perceived inability subtest, economic burden subtest, social burden subtest, time requirement subtest, and emotional burden subtest. The indicator's and our study are on the same direction.

In comparison of the mean scores of family burden assessment scale in terms of gender of athletes, it was found that three was not any significant difference but economic burden was more in females compared to males; that perceived inability was lower in females than that of males; and, when regarded in terms of time requirement, that the times dedicated to females was higher compared to the males. When the mean scores are evaluated in terms of social burden, physical burden, and emotional burden, it was found that they were close to each other.

Any significant difference was not found between the disability state, the number of years they do swimming sports, when disability state occurs, and age of athletes and sub factors of family burden assessment scale.

According to these results, the mothers taking points over 97 have burden. In the study we have carried out, when regarded to the general mean score of family load, the mean score was found 127 and it revealed that the families of swimmers had family burden.

\section{References}

Abelson, A. G. (1999). Respite care needs of parents of children with developmental disabilities. Focus on Autism \& Other Developmental Disabilities, 14(2), 96-100. https://doi.org/10.1177/108835769901400204

Chou, K. R. (2000) Caregiver Burden: A Concept Analysis, Journal of Pediatric Nursing, 15(6), 398-407. https://doi.org/10.1053/jpdn.2000.16709

Fishman, S., \& Wolf, L. (1991). The handicapped child: Psychological effects of parental, martial and sibling relations, Psychiatric Clinics of North America, 14(1).

Kaner, S. (2004). An examination of stress, social support and life satisfaction perceived by parents with disabilities. Ankara: Ankara University Scientific Research Project Final Report. Access date: 10.02.2014, http:/www.acikarsiv.edu.tr

Naomi, C., Isaacson, E. C., \& Julıe, A. N. (2014). Launching: The experiences of two young adults with intellectual disability and their families in transition to individual supported living. Journal of Intellectual \& Developmental Disability, 39(3), 270-281. https://doi.org/10.3109/13668250.2014.929643 
Notifier, F. (2014). The relationship between family burden and psychological endurance in families with children in need of special education. Golden Horn University Institute of Social Sciences psychology graduate program applied psychology graduate program. Istanbul

Öztürk, Y. (2011). Determination of needs and family burden of families with handicapped children Halic University Institute of Health Sciences Istanbul

Sar1, H. Y., Baser, G., \& Turan, J. M. (2006). Experiences of mothers of children with Down syndrome. Pediatric Nursing 18(4), 29-32. https://doi.org/10.7748/paed2006.05.18.4.29.c1024

Sarı, Y. H., \& Basbakkal, Z. (2008). Development of family burden assessment scale for families with mentally handicapped children. Ataturk University Journal of Nursing School, Erzurum, 11(3), 86-95.

Sivri, T. (2012). Determination of family stress, perceived social support and family burden of mothers with mental retardation. Abant İzzet Baysal University Special Education USA Graduate thesis.2012

The handicapped child: Psychological effects of parental, martial an disabling relations, Psychiatric Clinics of North America, 14(1).

\section{Copyrights}

Copyright for this article is retained by the author(s), with first publication rights granted to the journal.

This is an open-access article distributed under the terms and conditions of the Creative Commons Attribution license which permits unrestricted use, distribution, and reproduction in any medium, provided the original work is properly cited. 